Revista Brasileira de Odontologia Legal - RBOL

\title{
Antropologia forense
}

\section{ESTIMATIVA DE IDADE POR MEIO DO VOLUME DAS CÂMARAS PULPARES EM IMAGENS DE TOMOGRAFIA COMPUTADORIZADA DE FEIXE CÔNICO - REVISÃO DE LITERATURA.}

\section{Estimation of age using the volume of the pulp chambers in cone beam computed tomography images - a review.}

\author{
Priscila Dias PEYNEAU ${ }^{1}$, Thais Uenoyama DEZEM ${ }^{2}$. \\ 1. Radiologia Odontológica, Odontologia Legal, Odontologia, Universidade Vila Velha, Espírito Santo, Brasil \\ 2. Odontologia Legal, Odontologia do Trabalho, Odontologia, Faculdade São Leopoldo Mandic, São Paulo; Profa. de \\ Odontologia Legal e Clinica Integrada, Faculdade de Odontologia, Unicerrado, Goiatuba, Goiás, Brasil.
}

Informação sobre o manuscrito

Recebido em: 14 Abril 2020

Aceito em: 12 Maio 2020

\author{
Autor para contato: \\ Priscila Dias Peyneau. \\ Rua Chafic Murad 119, Vitória, ES, Brasil. 29050-660. \\ E-mail: priscila.peyneau@uvv.br.
}

\begin{abstract}
RESUMO
Introdução: A estimativa de idade é uma das formas de identificação na área forense que visa obter a idade mais próxima da idade cronológica em indivíduos vivos ou mortos. A análise por meio dos dentes é um dos métodos mais confiáveis, pois estes são considerados como os tecidos mais duros e resistentes do corpo humano. A idade em adultos pode ser estimada por meio da formação de dentina secundária com consequente redução do tamanho da câmara pulpar. O exame de tomografia computadorizada de feixe cônico (TCFC) é o único que contempla o volume pulpar e desta forma resulta em informações confiáveis e podem ser tomadas como um guia para predição de idade em adultos. Objetivo: revisar a literatura sobre o uso da TCFC como método de imagem para estimar a idade dental por meio do volume da polpa em dentes unirradiculares e multirradiculares. Resultados: A TCFC é o exame ideal para avaliar o volume da câmara pulpar, pois permite mensurar a distância vestíbulo-lingual e mésio-distal. Os dentes incisivos superiores se mostraram como ideais nesta avaliação pelo fato de serem unirradiculares. Considerações finais: A ciência forense pode usufruir desta tecnologia de imagem para estimar a idade nos casos periciais em indivíduos vivos ou mortos.
\end{abstract}

\section{PALAVRAS-CHAVE}

Odontologia legal; Determinação da idade pelos dentes; Tomografia computadorizada de feixe cônico; Antropologia forense.

\section{INTRODUÇÃO}

A identificação dos seres humanos é um conjunto de fatores e procedimentos técnicos, realizada por pessoas treinadas da área judicial ou policial, ou por profissionais com conhecimentos específicos na área biológica como médicos legistas e odontolegistas, que analisam e comparam características particulares dos indivíduos com o objetivo de individualizar uma pessoa ou um objeto ${ }^{1,2}$. Dentre alguns métodos de identificação utilizados como rotina incluem o reconhecimento visual por meio de vestimentas da vítima, de objetos pessoais, 
de impressões digitais, análises de DNA, exames de imagem, bem como análise dos fios de cabelo e dos dentes ${ }^{3}$.

A estimativa de idade é uma das formas de identificação na área forense que exige uma abordagem multidisciplinar visando obter a idade mais próxima da idade cronológica em indivíduos vivos ou mortos. O estudo dos dentes é um dos métodos mais confiáveis, pois estes são considerados como os tecidos mais duros do corpo humano, e possuem grande resistência à maioria dos efeitos ambientais, como incêndios, dessecação e decomposição, fatores genéticos, hormonais, nutricionais e patológicos quando comparados com as estruturas esqueléticas, sendo assim mais adequados para a estimativa de idade $e^{4-15}$.

Existem diversas técnicas para estimar a idade de um indivíduo, entretanto cada uma delas se refere a uma fase da vida. A estimativa da idade em adultos, quando o dente já está completamente formado, é motivo de grande dificuldade e então é necessária a análise de fatores regressivos ${ }^{4,10,12-14}$.

Várias técnicas foram relatadas para determinar a idade em populações adultas com por meio de métodos bioquímicos, histológicos e radiográficos. O método radiográfico apresenta vantagens em relação aos outros citados e resultam em informações confiáveis e podem ser tomadas como um guia para predição de idade em adultos ${ }^{6,10,12,14,16,17}$.

Os exames radiográficos bidimensionais, como a radiografia panorâmica e a periapical, podem ser utilizados para avaliar a relação do tamanho da área da câmara pulpar e do dente ${ }^{17-19}$. A principal desvantagem das radiografias é que elas estão sujeitas a ampliação e distorção, além de apresentarem sobreposição de estruturas. Portanto, uma avaliação simultânea das dimensões mésiodistal e vestíbulo-lingual dos dentes tem sido recomendada por ser mais acurada. Desta forma, a tomografia computadorizada é o método ideal e mais preciso para avaliar a relação volume de polpa/dente ${ }^{14,20}$

A tomografia computadorizada de feixe cônico (TCFC) representa um significativo avanço na radiologia e está ganhando espaço no campo das ciências forenses. Esta tecnologia oferece uma imagem tridimensional com indicação para tecidos mineralizados, especificamente na região dentomaxilofacial, permitindo a visualização das imagens nos planos axiais, coronais e sagitais $^{15,21}$. Além disso, possui vantagens em relação a limitação do campo de visão do feixe de raios $X$, a redução da dose de radiação, e a visualização dos dentes sem sobreposição, distorção ou ampliação $^{14,15}$. As imagens geradas permitem a obtenção de medidas angulares e lineares, além da facilidade na manipulação da imagem e qualidade da imagem $^{1,14,22}$.

O objetivo deste trabalho é descrever sobre o uso da TCFC como método de imagem para estimar a idade dental por meio do volume da polpa em dentes unirradiculares e multirradiculares.

\section{REVISÃO DE LITERATURA}

A estimativa de idade se destaca no cenário forense como informação vital para identificação. Devido às características de 
perenidade dos elementos dentais, os mesmos são considerados como peças chave para estimar a idade em indivíduos vivos ou mortos ${ }^{23}$.

Dentre os métodos de estimativa de idade em adultos descritos na literatura, podem-se destacar as medidas da razão entre a polpa e o dente, pois uma das mais importantes mudanças fisiológicas relacionadas à idade é a deposição de dentina secundária ${ }^{4,10,15}$. A dentina é composta por hidroxiapatita mineral (70\%), material orgânico $(20 \%)$ e água (10\%). Esta estrutura dental é mais dura que o osso, porém mais macia que o esmalte, e é composta principalmente de cristais de apatita fosfórica. Os odontoblastos, que iniciam a formação da dentina, estão localizados ao redor da câmara pulpar como uma única camada. Os íons cálcio da dentina e odontoblastos são conectados por canais no interior dos túbulos dentinários ${ }^{24}$. Existem dois tipos de dentina fisiológica: a dentina primária, que é depositada antes da formação da raiz estar completa, e a dentina secundária, que é formada após a coroa e a raiz estarem completas e em oclusão, e então continua a ser formada ao longo da vida resultando em diminuição progressiva do volume da cavidade pulpar com a idade. A formação da dentina secundária pode ser causada por atrição, abrasão, erosão, lesões cariosas, alterações na pressão na câmara pulpar, ou envelhecimento e consequentemente diminui $\mathrm{o}$ volume da câmara pulpar ${ }^{5,11-15,25}$.

Várias técnicas foram relatadas para determinar a idade em populações adultas com por meio de métodos bioquímicos e histológicos. Estes processos eram destrutivos, demorados e complexos, pois era necessário a extração dos dentes, o que torna inviável em indivíduos vivos $^{6,10,12,14,16,17}$.

Desta forma, os métodos de imagem ganharam relevância na área forense para estimar a idade por ser um método não destrutivo, ou seja, sem a necessidade de extração dental, e com bastante informação sobre os elementos dentais. Por meio dos exames imaginológicos é possível mensurar a polpa e então estimar a idade cronológica ${ }^{13,23}$.

Um dos primeiros estudos que se destacou neste tema, foi o método proposto por Kvaal et al. (1995) ${ }^{18}$, onde estimaram a idade a partir do tamanho da câmara pulpar em radiografias periapicais. Entretanto, deve-se considerar algumas características das radiografias que influenciam na avaliação de todo o volume da câmara pulpar. A primeira delas é o fato das radiografias serem bidimensionais não permitindo medidas no sentido vestíbulolingual da polpa. Outras duas considerações são: a ampliação e a distorção, que podem estimar medidas errôneas da região de interesse $\mathrm{e}^{13,14,20}$.

Estas desvantagens puderam ser eliminadas com a introdução da TCFC, pois esta técnica fornece imagens sem sobreposição com medidas precisas, sem distorção e ampliação. Além disso, a TCFC ganhou credibilidade por ser uma técnica de imagem onde os voxels são isotrópicos variando entre $0,4 \mathrm{~mm}$ e $0,075 \mathrm{~mm}$ permitindo diferenciar estruturas pequenas de diferentes densidades. Quanto menor o tamanho do voxel, maior é a resolução da imagem, e então as estruturas observadas 
são mais nítidas e podem ser distinguidas umas das outras devido a esta alta resolução espacial. Devido a isso, o uso de equipamentos com voxels menores é recomendada para determinar a anatomia interna dos canais radiculares, fraturas, reabsorções, bem como mensurar o volume da câmara pulpar ${ }^{12-14,26}$.

Para análise da câmara pulpar, que é o objetivo deste trabalho, esta estrutura necessita ser separada e detalhada para análise. Sendo assim, o exame de TCFC é importado para um software onde a estrutura de interesse será segmentada. A segmentação de imagem é importante para a identificação das estruturas, quantificação de volume, estudo anatômico e planejamento de tratamento. A técnica consiste na separação de uma área de todo o restante da imagem para obtenção de informações relevantes para o estudo em questão, mediante propriedades de descontinuidade e similaridade, como por exemplo, nível de cinza, e a definição precisa de sua extensão espacial. A precisão da segmentação determina o sucesso ou falha de um sistema de análise computadorizada $^{27,28}$.

O primeiro estudo que usou a TCFC para estimar a idade por meio do volume da câmara pulpar foi o de Yang et al., (2006) ${ }^{29}$. Foi um estudo piloto, onde os autores avaliaram 28 dentes unirradiculares pertencentes a 19 indivíduos, em imagens de TCFC de alta resolução por meio do aparelho 3DAccuitomo ( $J$ Morita Corporation, Osaka, Japan). Cada dente foi segmentado por meio de software para calcular o volume da polpa, e foi observada uma correlação moderada entre a relação volume pulpar e a idade biológica. Sendo assim, a técnica se mostrou promissora para a estimativa da idade dental de forma não invasiva, utilizando imagens de TCFC em indivíduos vivos. A partir deste momento, vários estudos foram realizados afim de estabelecer a correlação entre o volume da câmara pulpar e a estimativa de idade.

O volume da câmara pulpar de caninos da população indiana foi alvo de estudo por Jagannathan et al. $(2011)^{20}$. Foram avaliados por meio de TCFC, 188 caninos inferiores extraídos de indivíduos com idade entre 10 e 70 anos. $O$ aparelho utilizado foi 3DAccuitomo ( $\mathrm{J}$ Morita Corporation, Osaka, Japan) com voxel de $0,25 \mathrm{~mm}$ e para a segmentação foi usado o software Advantage Windows Workstation. Este software foi responsável por realizar a reconstrução da polpa e em seguida calcular o volume. Por meio do resultado puderam observar que a relação volume pulpar/dente é um indicador útil de idade, embora as correlações possam variar em diferentes populações e, portanto, fórmulas específicas devem ser aplicadas.

Star et al. $(2011)^{5}$ avaliaram a relação do volume da câmara pulpar em 111 exames de TCFC, sendo 57 do sexo feminino e 54 do sexo masculino, com idade entre 10 e 65 anos. Foram avaliados 214 dentes unirradiculares, dentre eles pode-se citar: incisivos, caninos e pré-molares superiores e inferiores. As imagens foram adquiridas no aparelho Scanora 3D (Soredex, Tuusula, Finlândia) com voxel entre 0,15 e $0,20 \mathrm{~mm}$ e cada dente/polpa foi segmentado pelo software Simplant ${ }^{\circledR}$ Pro Version 11.0 on Windows (Materialise 
Dental NV, Leuven, Belgium). O programa calcula automaticamente o volume das imagens tridimensionais do dente e da polpa. Após uma análise de regressão linear, observaram que o dente incisivo obteve maiores correlações com a idade. Além disso, foi relatado que as mulheres apresentaram forte relação entre 0 volume pulpar e a idade, mas não foi estatisticamente significante.

No estudo piloto de Pinchi et al. $(2015)^{30}, 148$ exames de TCFC de pacientes entre 10 e 80 anos de idade, feitos no aparelho Scanora 3D (Soredex, Tuusula, Finland), tiveram a câmara pulpar dos dentes incisivos centrais superiores avaliadas por meio do software Osirix $\circledast$ (OnDemand 3D software CyberMed Inc.). O volume foi calculado por meio de medidas lineares e áreas nas reconstruções sagitais e coronais de TCFC. Os resultados deste estudo mostram que a diminuição da câmara pulpar é um parâmetro confiável para estimar a idade dos adultos, mas não entre os sexos, e que a TCFC é uma abordagem fácil e não invasiva que permite o cálculo preciso do volume dos dentes. $O$ procedimento de validação no qual os volumes reais são comparados com aqueles calculados usando TCFC suporta a precisão da técnica realizada, e a boa concordância entre examinadores (ICC 0,99) demonstra que o método é altamente reprodutível.

$\mathrm{Ge}$ et al. $(2016)^{9}$ analisaram o volume da câmara pulpar em 115 exames de TCFC de mulheres e 125 de homens com idade entre 16 e 63 anos. As imagens foram adquiridas no tomógrafo NewTom VG (Quantitative Radiology, Verona, Italy) com voxel de $0,15 \mathrm{~mm}$ e então segmentadas por meio do software ITK-SNAP 2.4 para obtenção do volume. Todos os dentes superiores e inferiores foram selecionados para o estudo, exceto os terceiros molares e os primeiros pré-molares superiores. Dentre os 13 tipos de dentes avaliados, os segundos molares superiores apresentaram maior correlação do volume da câmara pulpar com a idade tanto no sexo feminino quanto no masculino, e o canino superior mostrou a menor correlação.

Foi realizado um estudo por Alsoleihat et al. $(2017)^{10}$ na população da Jordânia com idade entre 18 e 58 anos para investigar a estimativa da idade cronológica baseada em medidas do diâmetro da polpa na junção amelocementária do terceiro molar inferior. A amostra foi composta de 155 exames de TCFC (80 homens e 75 mulheres). O ICC entre os observadores foi excelente $(0,85$ e 0,87$)$ para as duas medidas, o que indica que o erro entre eles foi aleatório e estatisticamente insignificante. Os autores concluiram que a relação médiodistal da polpa/dente na região cervical não é um preditor confiável da idade cronológica em adultos, possivelmente devido à grande variabilidade em realação ao tamanho e formato deste dente e também ao tempo de desenvolvimento dos mesmos.

Nos estudos de Bing et al. $(2017)^{11}$ com 103 exames de TCFC (51 homens 52 mulheres), a câmara pulpar de caninos superiores na população chinesa foi analisada por meio do software Mimics (Materialise NV, Belgium). Este software foi utilizado para a segmentação e reconstrução tridimensional da cavidade pulpar. Uma análise de regressão linear foi aplicada com as variáveis e permitiu que a 
razão entre o volume da polpa e o volume do dente pudesse ser usada para inferir a idade do indivíduo. Desta forma, a técnica pode fornecer um novo método e caminho para o reconhecimento individual na odontologia forense.

Biuki, Razi, Faramarzi $(2017)^{12}$ avaliaram o volume da câmara pulpar, por meio do software Mimics (Materialise NV, Belgium), em 122 exames de TCFC, onde 46 eram do sexo masculino e 76 do feminino, com idade entre 13 e 70 anos. Os dentes anteriores superiores e inferiores, em um número de 732 dentes, foram a amostra selecionada. O tomógrafo utilizado foi o NewTom VGi (QR srl, Verona, Italy) e o tamanho do voxel foi de $0,15 \mathrm{~mm}$. Cada dente foi segmentado e reconstruído em formato tridimensional. Para uso do software o operador utiliza ferramentas disponíveis para remover toda a estrutura dental, deixando apenas a polpa, e então o volume é calculado automaticamente. O resultado do estudo mostrou correlação inversa entre a idade e o volume da câmara pulpar, ou seja, quando o volume foi menor a idade foi maior. Foi observado que no sexo masculino e nos dentes incisivos centrais e caninos, a correlação foi mais forte. Verificou-se ainda, que a média do volume dos dentes anteriores teve correlação ainda mais forte quando comparado com o valor de um único dente.

Em um estudo na população turca, Aydin \& Bayrak (2018) ${ }^{13}$ avaliaram se o volume da câmara pulpar dos incisivos centrais superiores apresentava correlação com a idade cronológica. Foram avaliados 120 exames de TCFC (60 homens e 60 mulheres) com idade entre 14 e 75 anos de idade. Os exames foram realizados no aparelho I-CAT 3D Imaging System (Imaging Sciences International, Hatfield, PA) e as medidas da área da polpa/dente foram obtidas pelo software inVivo 5 (Anatomage, San Jose, CA). As bordas da polpa e dos dentes foram desenhados manualmente. As bordas foram determinadas usando um mínimo de 10 pontos para a polpa e pelo menos 20 pontos para o dente, e a área sob esses pontos de orientação foi calculada. Os resultados mostraram que a área da polpa dental dos incisivos centrais superiores é um método confiável para estimativa de idade na população turca. Além disso, o ICC entre os avaliadores se mostrou excelente $(0,869)$ demosntrando que as medidas podem ser reproduzidas.

Gulsahi et al. (2018) ${ }^{14}$ avaliaram o volume da câmara pulpar de incisivos centrais, laterais e caninos superiores e caninos e pré-molares inferiores. Foram coletados exames de TCFC de pacientes acima de 15 anos de idade, pertencentes a um banco de dados. A quantidade total da amostra foi de 655 dentes referente a 204 pacientes (108 homens e 96 mulheres). As imagens foram adquiridas no aparelho Kodak CS9300 (Carestream Health, Rochester, NY), e o tamanho do voxel variou entre 0,09 e 0,2 $\mathrm{mm}$. A segmentação foi feita no software 3D DOCTOR (Able Software Corp, Lexington, MA) que calcula de forma automática o valor do volume da câmara pulpar. Este software permite a segmentação dos tecidos em cortes axiais e sagitais consecutivos, permitindo a visualização da polpa em cada nível do dente, desde o ápice até a coroa. Isso 
permite uma segmentação detalhada das bordas do dente, corte a corte, de forma manual com o uso do mouse. Em seguida o cálculo do volume da polpa e do dente é realizado pelo programa. A correlação entre idade e volume da câmara pulpar se apresentou mais forte no incisivos central superior, e entre os sexos não houve correlação. O ICC entre os avaliadores foi de 0,81-0,90, considerado como excelente, demosntrando que a técnica é reproduzível.

Asif et al. (2019) ${ }^{15}$ investigaram a associação entre idade cronológica e volume da câmara pulpar na população da Malásia e da China utilizando imagens de TCFC. A amostra foi de 300 exames, sendo 179 malaios e 121 chineses, com idade entre 16 e 65 anos, e os dentes selecionados foram os incisivos centrais superiores direito e os caninos superiores direito e esquerdo. $O$ aparelho utilziado para aquisição da imagem foi o i-CAT Cone Beam 3D Dental Imaging System (Imaging Sciences International, Hatfield, USA) com voxel de $0,3 \mathrm{~mm}$. As imagens em DICOM foram importadas para o software Mimics (Materialise NV, Belgium) onde foi feita a segmentação e o cálculo do volume da câmara pulpar. O resultado mostrou que não houve diferença significante entre os malaios e os chineses, e houve forte correlação para o incisivo central superior direito. O ICC entre os avaliadores foi 0,968 , considerado como excelente, demosntrando a reprodutibilidade da técnica.

\section{DISCUSSÃO}

Os dentes são bastante úteis na identificação humana e estimativa de idade, pois podem permanecer intactos e suportar condições adversas (mecânicas, térmicas e químicas) anos após a morte do indivíduo. Ao longo da existência do indivíduo, eles sofrem alterações biológicas, patológicas e são submetidos a procedimentos terapêuticos que os tornam identificáveis em cadáveres desconhecidos ${ }^{5-9,13-15}$.

A dentina que compõe a camada interna do dente sofre alterações ao longo do tempo impactando diretamente no tamanho da polpa dental. A formação da dentina secundária, que promove a diminuição do volume da câmara pulpar, pode ser causada por atrição, abrasão, erosão, lesões cariosas ou envelhecimento, o que favorece a estimativa de idade em pacientes adultos ${ }^{5,11-15,25}$.

Desta forma, vários estudos a respeito da estimativa de idade por meio da análise volumétrica da câmara pulpar com imagens de TCFC tem sido realizados em várias populações do mundo. Além disso, em cada relato se observa o estudo com um tipo de dente e com técnicas diferentes, permitindo a variabilidade de resultados entre os artigos publicados.

A introdução da TCFC para avaliar estruturas tridimensionais é uma ferramenta que tem se destacado na ciência forense para identificação humana e para estimar a idade de indivíduo. Uma grande vantagem desta tecnologia de imagem é que o método não é invasivo, permitindo o uso em pacientes vivos. Desta forma, o volume da câmara pulpar, que é uma estrutura tridimensional, pode ser avaliado em imagens de TCFC por meio de medidas precisas, sem distorção e ampliação. Existem diversos modelos e marcas de aparelho de TCFC, bem como vários 
tamanhos de voxel. Na revisão de lieratura realizada, se observa que os voxels puderam variar entre 0,09 e $0,3 \mathrm{~mm}^{5,9,12-}$ ${ }^{15,20,30}$. Quando se tem tamanhos de voxels variados, isso pode resultar em diferentes mensurações volumétricas de uma mesma estrutura anatômica. De acordo com a literatura, voxels com tamanho entre 76 e $200 \mu \mathrm{m}$ não apresentam alterações significativas em relação a medidas volumétricas. Entretanto quando o tamanho ultrapassa $300 \mu \mathrm{m}$, o que equivale a 0,3 $\mathrm{mm}$, a subestimação das medidas já é significante ${ }^{26}$. Dentre os trabalhos relatados, apenas Asif et al. (2019) ${ }^{15}$ utilizou voxel de $0,3 \mathrm{~mm}$. Este tamanho de voxel ainda é aceitável para uma análise volumétrica confiável, principalmente se os elementos dentais da amostra forem unirradiculares. Normalmente, os dentes anteriores são mais fáceis de serem segmentados devido a amplitude da câmara pulpar e canal radicular, o que torna a reconstrução $3 \mathrm{D}$ mais rápida.

O tamanho do voxel influencia também na qualidade da segmentação da área escolhida para avaliação ${ }^{26}$. Isso ocorre porque a segmentação avalia o conteúdo dos voxels/pixels da imagem, e então na região de interesse é incluído voxels/pixels com as mesmas propriedades na mesa escala de cinza ${ }^{28}$. A segmentação pode ser feita de três formas: por meio da determinação do contorno da estrutura de interesse feita pelo operador, ou seja, manualmente; de forma automática, quando o software escolhe os voxels/pixels baseado em regras predeterminadas; ou semiautomática, quando os métodos de segmentação manual e automática são associados, isto é, automatizando parte do processo e deixando que o operador faça ajustes de acordo com ajude com a sua experiência. Qualquer falha ainda inserida pelo processo de segmentação automática pode ser ajustada manualmente ${ }^{27,28}$. O processo manual pode minimizar alguns problemas, como por exemplo, a delimitação do terço apical dos dentes. Esta porção é mais fina e gera dificuldades na segmentação devendo ser feito de forma cautelosa para que dados não sejam perdidos. Outro exemplo é quando há calcificações no interior da câmara pulpar, o que impede a leitura correta pelo software. Devido a isso, os resultados dos estudos devem ser interpretados com cuidado, pois o volume pode não ter sido obtido em sua totalidade $^{15}$.

Diversos softwares estão disponíveis no mercado para realizar a segmentação de estruturas anatômicas. A maioria dos autores relatados nesta revisão de literatura lançaram mão de softwares semiautomáticos para o cálculo do volume da câmara pulpar, como por exemplo o Advantage Windows Workstation, inVivo 5, ITK-snap 2.4, Mimics 16.0, Simplant $\AA^{5,9,13,20}$. De acordo com o que descreve a literatura, se observa que a forma semiautomática é mais interessante, pois permite um ajuste do operador a fim de determinar de forma mais fiel a estrutura a ser analisada. O contraste e a nitidez das imagens obtidas por meio de aparelho de TCFC ainda precisam ser melhorados para facilitar a segmentação. Além disso, o método precisa ser mais rápido e fácil para ser aplicado na prática clínica $^{26}$. 
O índice de correlação intraclasse (ICC) que avalia a confiabilidade do estudo ser reproduzido por meio das medidas foi considerado excelente de acordo com a maioria das publicações deste tipo de estudo. O ICC variou de 0,81 a 0,99, o que mostra a confiabilidade na reprodução do método $^{10,13-15,30}$.

Nos estudos citados, se percebe que vários dentes foram utilizados para a análise, tanto unirradiculares quanto multirradiculares. Dentre os dentes escolhidos, pode-se citar: incisivos, caninos, pré-molares, molares superiores e inferiores, exceto terceiros molares superiores $^{5,9-15,30}$. Os incisivos superiores foram os dentes que apresentaram maiores correlações com a idade ${ }^{5,12-15,30}$, por serem mais fáceis de serem segmentados devido ao canal radicular ser mais amplo $e$ consequentemente possibilitarem menor erro no resultado do volume pulpar. Somente um estudo mostrou correlação mais forte entre um dente multirradicular e a idade ${ }^{9}$.

Em relação ao sexo, foi observado no estudo de Star et al. $(2011)^{5}$ uma forte correlação entre o volume pulpar e a idade nas mulheres, mas não foi estatisticamente significante. Já no relato de Biuki, Razi, Faramarzi $(2017)^{12}$, o sexo masculino apresentou correlação estatisticamente significante em relação ao volume da câmara pulpar. Em outros estudos não houve diferença estatisticamente significante do volume da câmara pulpar em relação aos sexos feminino e masculino ${ }^{9,14}$. Observa-se que o volume pulpar não é o melhor indicativo para dimorfismo sexual. Vale ressaltar que pode haver mudanças das características sexuais $m$ relação a etnia, localização geográfica e hábitos alimentares de cada população.

Uma das limitações em relação ao uso da TCFC para a estimativa de idade em pacientes vivos é a dose de radiação emitida. Apesar do exame fornecer imagens tridimensionais precisas da anatomia dental favorecendo a análise do volume pulpar, deve-se ressaltar que a dose efetiva é maior em TCFC do que em radiografias panorâmicas. Entretanto, esta dose pode variar de acordo com o tamanho da área irradiada, com o aparelho utilizado e com os parâmetros utilizados na exposição $^{14,15,21,31,32}$. Vale ressaltar que em exames de TCFC maiores informações são colhidas para análise post mortem e em vivos obtendo assim benefício na área forense. Para isso, deve-se utilizar a menor dose possível para diagnóstico orientando a indicação específica para cada caso de acordo com o princípio de ALADAIP (As Low as Diagnostically Acceptable being Indication-oriented and Patient-specific) ${ }^{33}$.

\section{CONSIDERAÇÕES FINAIS}

Conclui-se que a tomografia computadorizada de feixe cônico (TCFC) é um método confiável para estimar a idade por meio do volume pulpar, pois permite mensurar a distância vestíbulo-lingual e mésio-distal.

Os incisivos superiores se mostraram como ideais nesta avaliação pelo fato de serem unirradiculares e então facilitar a segmentação da imagem para o cálculo do volume. Desta forma, a ciência forense pode usufruir desta tecnologia de 


\begin{abstract}
Introduction: The age estimate is one of the forms of identification in the forensic area that aims to obtain the age closest to the chronological age in living or dead individuals. Teeth analysis is one of the most reliable methods, as these are considered to be the hardest and most resistant tissues of the human body. Adult age can be estimated through the formation of secondary dentin, with a consequent reduction in the size of the pulp chamber. The conical beam computed tomography (CBCT) exam is the only one that contemplates the pulp volume and thus results in reliable information and can be taken as a guide for predicting age in adults. Objective: review the use of CBCT as an imaging method to estimate dental age through pulp volume in uniradicular and multiradicular teeth. Results: CBCT is the ideal test to assess the volume of the pulp chamber, as it allows the measurement of the buccal-lingual and mesio-distal distance. The upper incisor teeth were shown to be ideal in this evaluation because they are uniradicular. Final considerations: Forensic science can take advantage of this imaging technology to estimate age in expert cases in living or dead individuals.
\end{abstract}

\title{
KEYWORDS
}

Forensic dentistry; Age determination by teeth; Cone-beam computed tomography; Forensic anthropology.

\section{REFERÊNCIAS}

1. Carvalho SPM, Silva RHA, Lopes Jr C, Sales-Peres A. A utilização de imagens na identificação humana em odontologia legal. Radiol Bras. 2009; 42(2):125-30.

2. Vanrell JP. Odontologia legal e antropologia forense. Rio de Janeiro: Rio de Janeiro; 2012.

3. Gruber J, Kameyama MM. O papel da radiologia em odontologia legal. Pesqui Odontol Bras. 2001; 15(3):263-68.

4. Silva RF, Marinho DEA, Botelho TL, Caria THF, Bérzin F, Daruge Júnior E. Estimativa da idade por meio de análise radiográfica dos dentes e da articulação do punho: relato de caso pericial. Arq Odontol. 2008; 44(2):93-8.

5. Star $\mathrm{H}$, Thevissen $\mathrm{P}$, Jacobs $R$, Fieuws $\mathrm{S}$, Solheim T, Willems G. Human dental age estimation by calculation of pulp-tooth volume ratios yielded on clinically acquired cone beam computed tomography images of monoradicular teeth. J Forensic Sci. 2011; 56(1):S77-82. https://doi.org/10.1111/j.15564029.2010.01633.x.

6. Sakuma A, Saitoh $H$, Suzuki $Y$, Makino $Y$, Inokuchi G, Hayakawa $M$, et al. Age estimation based on pulp cavity to tooth volume ratio using postmortem computed tomography images. J Forensic Sci. 2013; 58(6):1531-35.

7. Park K, Ahn J, Kang S, Lee E, Kim S, Park $S$, et al. Determining the age of cats by pulp cavity/tooth width ratio using dental radiography. J Vet Sci. 2014; 15(4):557-61. https://doi.org/10.1111/1556-4029.12175.

8. Albuquerque Neto $A D$, Farias Neto $A M$, Cavalcante JRD, Cavalcante DKF, Sampaio TRC, Costa VS. Efeito das altas temperaturas aos tecidos bucodentais e materiais odontológicos: revisão de literatura. Rev Bras Odontol Leg. 2015;
2(2):89-104 .

http://dx.doi.org/10.21117/rbol.v2i2.28.

9. Ge ZP, Yang P, Li G, Zhang JZ, Ma XC. Age estimation based on pulp cavity/chamber volume of 13 types of tooth from cone beam computed tomography images. Int J Legal Med. 2016; 130(4):1159-67.

http://dx.doi.org/10.1007/s00414-016-13846.

10. Alsoleihat $\mathrm{F}$, Al-Shayyab $\mathrm{MH}$, Kalbouneh $\mathrm{H}$, Al-Zer H, Ryalat S, Alhadidi A et al. Age prediction in the adult based on the pulp-totooth ratio in lower third molars: A Conebeam CT study. Int J Morphol. 2017; 35(2):488-93.

http://dx.doi.org/10.4067/S071795022017000200017.

11. Bing L, Wu XP, Hong-shangguan; Xiao W, Yun KM. Morphology and volume of maxillary canine pulp cavity for individual age estimation in forensic dentistry. Int $\mathrm{J}$ Morphol. 2017; 35(3):1058-62. http://dx.doi.org/10.4067/S071795022017000300039.

12. Biuki N, Razi T, Faramarzi M. Relationship between pulp-tooth volume ratios and chronological age in different anterior teeth on CBCT. J Clin Exp Dent. 2017; 9(5): e688-e693.

http://dx.doi.org/10.4317/jced.53654.

13. Aydın ZU, Bayrak S. Relationship Between Pulp Tooth Area Ratio and Chronological Age Using Cone-beam Computed Tomography Images. J Forensic Sci. 2019; 64(4):1096-99.

http://dx.doi.org/10.1111/1556-4029.13986

14. Gulsahi A, Kulah CK, Bakirarar B, Gulen O, Kamburoglu K. Age estimation based on pulp/tooth volume ratio measured on conebeam CT images. Dentomaxillofac Radiol. 2018; 47(1):20170239. http://dx.doi.org/10.1259/dmfr.20170239. 
15. Asif MK, Nambiar P, Mani SA, Ibrahim NB, Khan IM, Lokman NB. Dental age estimation in Malaysian adults based on volumetric analysis of pulp/tooth ratio using CBCT data. Leg Med (Tokyo). 2019; 36:508.

https://doi.org/10.1016/j.legalmed.2018.10. $\underline{005}$.

16. Niquini BTB, Villalobos MIOB, Manzi FR, Bouchardet $\mathrm{FCH}$. Necessidade de estimativa da idade pelos dentes em processo civil de indenização - relato de caso pericial. Rev Bras Odontol Leg RBOL. 2015; 2(2):116-25. http://dx.doi.org/10.21117/rbol.v2i2.35.

17. Gotmare SS, Shah T, Periera T, Waghmare MS, Shetty S, Sonawane S, et al. The coronal pulp cavity index: A forensic tool for age determination in adults. Dent Res J (Isfahan). 2019; 16(3):160-65.

18. Kvaal SI, Kolltveit KM, Thomsen IO, Solheim T. Age estimation of adults from dental radiographs. Forensic Sci Int. 1995; 74(3):175-85

http://dx.doi.org/10.1016/03790738(95)01760-g.

19. Jain $S$, Nagi $R$, Daga $M$, Shandilya $A$, Shukla A, Parakh A, et al. Tooth coronal index and pulp/tooth ratio in dental age estimation on digitalpanoramic radiographs-A comparative study. Forensic Sci Int. 2017; 277:115-21. http://dx.doi.org/10.1016/j.forsciint.2017.05. $\underline{006}$.

20. Jagannathan $N$, Neelakantan $P$, Thiruvengadam C, Ramani $\mathrm{P}$, Premkumar $P$, Natesan $A$, et al. Age estimation in a Indian population using pulp/tooth volume ratio of mandibular canines obtained from cone beam computed tomography. J Forensic Odontostomatol. 2011; 1(29):1-6.

21. Cotton TP, Geisler TM, Holden DT, Schwartz SA, Schindler WG. Endodontic applications of cone-beam volumetric tomography. J Endod. 2007; 33(9):112132. http://dx.doi.org/10.1016/j.joen.2007.06.011

22. Garib DG, Raymundo Junior R, Raymundo MV, Raymundo DV, Ferreira SN. Tomografia computadorizada de feixe cônico (Cone beam): entendendo este novo método de diagnóstico por imagem com promissora aplicabilidade na Ortodontia. Rev Dent Press Ortodon Ortop Facial. 2007; 12(2):139-56. https://doi.org/10.1590/S141554192007000200018.

23. Gadelha MNV, Lima JCA, Ribeiro ILA, Santiago BM. Aplicabilidade do volume da câmara pulpar para a estimativa de idade em adultos a partir de tomografias computadorizadas de feixe cônico: um estudo piloto. Rev Bras Odontol Leg
RBOL. 2019; 6(1):30-39.

http://dx.doi.org/10.21117/rbol.v6i1.240.

24. Chun K, Choi H, Lee J. Comparison of mechanical property and role between enamel and dentin in the human teeth. $J$ Dent Biomec. 2014; 5: 1758736014520809. http://dx.doi.org/10.1177/17587360145208 $\underline{09}$.

25. Drusini AG. The coronal pulp cavity index: a forensic tool for age determination in human adults. Cuad Med Forense. 2008; 14(53-54):235-49.

26. Maret D, Telmon N, Peters OA, Lepage B, Treil J, Inglèse $\mathrm{JM}$, et al. Effect of voxel size on the accuracy of $3 D$ reconstructions with cone beam CT. Dentomaxillofac Radiol. 2012; 41(8):649-55. http://dx.doi.org/10.1259/dmf/81804525.

27. Faria DAB. Segmentação, Reconstrução e Quantificação 3D de Estruturas em Imagens Médicas - Aplicação em Imagem Funcional e Metabólica. Dissertação (Mestrado). Faculdade de Engenharia da Universidade do Porto, Porto; 2013. 81p.

28. Costa ALF, Yasuda CL, Nanhás-Scocate ACR. Utilização de softwares livres para visualização e análise de imagens 3D na Odontologia. Rev Assoc Paul Cir Dent. 2016; 70(1):76-81

29. Yang $F$, Jacobs R, Willems G. Dental age estimation through volume matching of teeth imaged by cone-beam CT. Forensic Sci Int. 2006; 159(15):S78-S83. http://dx.doi.org/10.1016/j.forsciint.2006.02. $\underline{031}$.

30. Pinchi V, Pradella F, Buti J, Baldinotti C, Focardi M, Norelli GA. A new age estimation procedure based on the $3 D$ CBCT study of the pulp cavity and hard tissues of the teeth for forensic purposes: a pilot study. J Forensic Leg Med. 2015; 36:150-57. http://dx.doi.org/10.1016/j.jflm.2015.09.015.

31. Eliášová H, Dostálová T. 3D Multislice and Cone-beam Computed Tomography Systems for Dental Identification. Prague Medical Report 2017; 118(1): 14-25.

32. Shin HS, Nam KC, Park H, Choi HU, Kim HY, Park CS. Effective doses from panoramic radiography and CBCT (cone beam CT) using dose area product (DAP) in dentistry. Dentomaxillofac Radiol 2014; 43(5):20130439. http://dx.doi.org/10.1259/dmfr.20130439.

33. Oenning AC, Jacobs R, Pauwels R, Stratis A, Hedesiu M, Salmon B, Dimitra Research Group. Cone-beam CT in paediatric dentistry: DIMITRA project position statement. Pediatr Radiol. 2018;48(3):30816. http://dx.doi.org/0.1007/s00247-0174012-9. 